Enferm Bras 2020;19(1):35-41

https://doi.org/10.33233/eb.v19i1.3366

\title{
ARTIGO ORIGINAL \\ O perfil epidemiológico das gestantes notificadas com sífilis no município do Rio de Janeiro - 2008 a 2017
}

Luiz Alberto de Freitas Felipe*, Clarissa Coelho Vieira Guimarães**, Vanessa Oliveira Ossola da Cruz $^{* * *}$, Michelle Freitas de Souza ${ }^{* \star *}$, Roberto Carlos Lyra da Silva, D.Sc. ${ }^{* * * *}$, Beatriz Gerbassi Costa Aguiar, D.Sc. ${ }^{* * \star \star * *}$

*Enfermeiro, Mestrando em Enfermagem, Universidade Federal do Estado do Rio de Janeiro, Rio de Janeiro/RJ, Enfermeiro da Estratégia de Saúde da Família do Município do Rio de Janeiro, ${ }^{*}$ Enfermeira, Mestranda em Enfermagem, Universidade Federal do Estado do Rio de Janeiro,

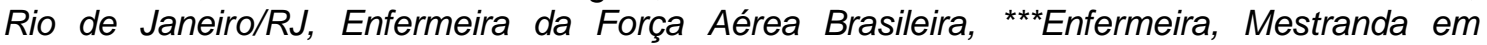
Enfermagem, Universidade Federal do Estado do Rio de Janeiro, Rio de Janeiro/ RJ, ****Enfermeira, Especialista em Saúde da Família, Universidade Gama Filho, Rio de Janeiro/RJ, Enfermeira Federal do Hospital Antonio Pedro, ${ }^{* * * * * E n f e r m e i r o, ~ P r o f e s s o r ~ A s s o c i a d o ~ I I ~ d a ~}$ Universidade Federal do Estado do Rio de Janeiro, Rio de Janeiro/RJ, ${ }^{* * * \star * \star}$ Enfermeira, Professora associada I da Universidade Federal do Estado do Rio de Janeiro

Recebido em 10 de novembro de 2019; aceito em 19 de fevereiro de 2020.

Correspondência: Luiz Alberto de Freitas Felipe, Avenida Tenente Coronel Muniz de Aragão, 1625 - Bloco 3/902, 22.765-007 Rio de Janeiro RJ

Luiz Alberto de Freitas Felipe: enfermeiroluizalbertodefreitas@gmail.com

Clarissa Coelho Vieira Guimarães: clarissaknog@hotmail.com

Vanessa Oliveira Ossola da Cruz: vanessa.ossola.cruz@gmail.com

Michelle Freitas de Souza: michelle.freitas@oi.com.br

Roberto Carlos Lyra da Silva: proflyra@gmail.com

Beatriz Gerbassi Costa Aguiar: nildo.ag@terra.com.br

\section{Resumo}

Objetivou-se conhecer incidência da sífilis em gestantes no município do Rio de Janeiro e descrever o perfil epidemiológico dos casos de gestantes com sífilis notificadas nesse município. Estudo epidemiológico, retrospectivo com abordagem quantitativa. A coleta de dados secundários foi obtida pela base de dados do Sistema de Informação e Agravos de Notificação - SINAN, disponibilizados pelo TABNET - RIO, o período analisado foi de 2008 a 2017. Os resultados mostraram que no período registraram-se 22.085 casos de sífilis em gestantes. A taxa de incidência variou entre 5.31 a 58,87/1.000 nascidos vivos. O estudo revelou que $63,75 \%$ das gestantes notificadas eram pretas ou pardas, com baixa escolaridade $(44,28 \%)$ e faixa etária entre 15 e 24 anos, 39,32\% das gestantes foram diagnosticadas com sífilis no primeiro trimestre de gestação, e a maioria realizou o tratamento de acordo com protocolo do Ministério da saúde, quanto ao parceiro, apenas $32,66 \%$ realizam o tratamento concomitante a gestante. O número de casos notificados é crescente, sugerindo que há uma necessidade de melhoria na assistência ao pré-natal, diagnóstico precoce e tratamento adequado.

Palavras-chave: sífilis, gestantes, cuidado pré-natal.

\section{Abstract}

Epidemiological profile of pregnant women reported with syphilis in the municipality of Rio de Janeiro - 2008 to 2017

The objective of this study was to know the incidence of syphilis in pregnant women in the city of Rio de Janeiro and to describe the epidemiological profile of pregnant women with syphilis. Epidemiological, retrospective study with a quantitative approach. Secondary data collection was obtained through the SINAN Information System and Notification Diseases Database, made available by TABNET - RIO. The period analyzed was from 2008 to 2017. The results showed that in the period, there were 22,085 cases of syphilis in pregnant women. The incidence rate ranged from 5.31 to $58.87 / 1,000$ live births. The study revealed that $63.75 \%$ of pregnant women were black or brown, with low schooling (44.28\%) and age group between 15 and 24 years, 39.32\% 
of women were diagnosed with syphilis in the first trimester of gestation, and most of them undergo treatment according to the protocol of the Ministry of Health. Regarding the partner, only $32.66 \%$ carry out concomitant treatment with the pregnant woman. The number of reported cases is increasing, suggesting that there is a need for improvement in prenatal care, early diagnosis and appropriate treatment.

Keywords: syphilis, pregnant women, prenatal care.

\section{Resumen}

\section{Perfil epidemiológico de mujeres embarazadas notificadas con sífilis en el municipio de} Rio de Janeiro, 2008 a 2017

Este estudio tuvo como objetivo conocer la incidencia de sífilis en mujeres embarazadas en la ciudad de Río de Janeiro y describir el perfil epidemiológico de los casos de mujeres embarazadas con sífilis reportados en la ciudad de Río de Janeiro. Estudio epidemiológico retrospectivo con abordaje cuantitativo. La recolección de datos secundarios se obtuvo de la base de datos del Sistema de Información SINAN y de Informes de Trastornos de Informes, disponible en TABNET - RIO, de 2008 a 2017. Los resultados mostraron que en el período hubo 22,085 casos de sífilis en mujeres embarazadas. La tasa de incidencia varió de 5.31 a 58.87 / 1,000 nacimientos vivos. El estudio reveló que el $63.75 \%$ de las mujeres embarazadas notificadas eran negras o marrones, con baja educación (44.28\%) y con edades entre 15 y 24 años, el $39.32 \%$ de las mujeres embarazadas son diagnosticadas con sífilis en el primer trimestre del embarazo, y la mayoría realiza el tratamiento de acuerdo con el protocolo del Ministerio de Salud, en cuanto a la pareja, solo el $32.66 \%$ realiza el tratamiento concomitante con mujeres embarazadas. El número de casos reportados está aumentando, lo que sugiere que existe la necesidad de mejorar la atención prenatal, el diagnóstico temprano y el tratamiento adecuado.

Palabras-clave: sífilis, mujeres embarazadas, cuidados prenatales.

Introdução

A sífilis é uma doença sexualmente transmissível causada pela bactéria treponema pallidum e, hoje, ainda se caracteriza como um grande desafio à saúde pública de todo o mundo. É uma doença transmitida por via sexual, vertical e raramente por via indireta [1].

De acordo com a via de transmissão, a sífilis é classificada em adquirida ou sífilis congênita. A sífilis adquirida é de transmissão predominantemente sexual e pode ser primária, secundaria terciária ou latente, a sífilis congênita o treponema pallidum, presente na corrente sanguínea da gestante infectada, atravessa a barreira placentária e penetra na corrente sanguínea do feto [2].

A sífilis congênita permanece como um grave problema de saúde pública no Brasil e em outros países da América Latina. Dados nacionais revelam a ocorrência de 54.141 casos de sífilis congênita no período de 2000 a 2010, com uma incidência, em 2009, de 1,7 casos por 1.000 nascidos vivos no país. Considerando os resultados do último estudo sentinela realizado em 2004, que encontrou uma prevalência de sífilis na gestação de 1,6\%, estima-se uma subnotificação de $67 \%$ dos casos de Sífilis congênita a cada ano, indicando uma situação ainda mais grave [1].

No ano de 2016, foram notificados 37.436 casos de sífilis em gestantes e 20.474 casos de sífilis congênita entre eles, 185 óbitos no Brasil. A maior proporção dos casos foi notificada na região Sudeste. Quando observadas as taxas, individualmente para cada estado, destacamse as elevadas taxas de sífilis em gestantes encontradas no Espírito Santo, seguido do estado do Rio de Janeiro. Em relação à sífilis congênita, estes estados permanecem em evidência, ao lado do estado de Pernambuco. Quando observados os óbitos por sífilis congênita em menores de 1 ano de idade, sobressai a taxa de 18,1 óbitos/ 1.000 nascidos vivos no estado do Rio de Janeiro, representando $23,2 \%$ do total observado em todo o país [3].

No município do Rio de Janeiro a situação também permanece grave, entre janeiro de 2008 e outubro de 2013, foram notificados 6.754 casos de sífilis em gestantes. A taxa de detecção da doença em gestante no município em 2012 foi de 22,7 casos por 1.000 nascidos vivos, se compararmos as taxas de detecção de 2008 a 2012, observa-se um aumento de aproximadamente $280 \%$. Os bairros que apresentaram maior taxa de detecção neste período foram Madureira e adjacências, seguido do bairro da Leopoldina [4].

As fragilidades no manejo do tratamento adequado da sífilis na gestação estão relacionadas às consultas de pré-natal com início tardio, na quebra da continuidade do cuidado com mudança de unidade de saúde durante a assistência, dificuldades no diagnóstico da sífilis 
durante a gestação, falhas no tratamento da gestante e, principalmente, do parceiro; além de falta de orientações sobre a doença e sobre uso de preservativos [1].

Baseado nos dados apresentados é possível compreender que embora a sífilis seja uma doença para a qual existem recursos diagnósticos e terapêuticos simples e de baixo custo, seu controle na gestação mostra-se um desafio para os gestores e para os profissionais de saúde. Isso em decorrência do curto intervalo da gestação para a realização do seu diagnóstico e tratamento, pela dificuldade de abordagem das doenças sexualmente transmissíveis, principalmente durante a gestação, e provavelmente pelo desconhecimento da magnitude desse agravo e dos danos que ele pode causar à saúde da mulher e do bebê pela população e pelos profissionais de saúde [5].

Diante do exposto, este estudo tem como objetivo conhecer a incidência da sífilis em gestantes no município do Rio de Janeiro e descrever o perfil epidemiológico dos casos de gestantes com sífilis notificadas no Município do Rio de Janeiro.

O estudo traz abordagem para reflexão do profissional de saúde que realiza consultas de pré-natal, para a segurança e a qualidade na assistência prestada orientada pelos direitos e baseada em evidências, a qual constitui uma importante estratégia na busca da promoção a saúde e dos direitos sexuais e reprodutivos das mulheres.

\section{Material e métodos}

Trata-se de um estudo epidemiológico, retrospectivo com abordagem quantitativa. A coleta de dados secundários foi obtida pela base de dados do Sistema de Informação e Agravos de Notificação (SINAN), disponibilizados pelo TABNET - RIO, o período analisado foi 2008 a 2017.

O estudo epidemiológico é definido como o estudo da distribuição e dos determinantes das doenças ou condições relacionadas à saúde em populações especificadas, o estudo inclui vigilância, observação, pesquisa analítica e experimento [6].

O estudo foi realizado no município do Rio de Janeiro capital do estado de Rio de Janeiro, localizado na região sudeste do Brasil. A coleta de dados foi realizada no site do DATA SUS/ TABNET em janeiro 2019, considerando as gestantes notificadas com sífilis no período de 2008 a 2017 no município do Rio de Janeiro.

Não houve necessidade do envio da pesquisa para plataforma Brasil, considerando que o estudo foi realizado com dados secundários de acesso livre, disponíveis na base de dados do Sistema de Informação e Agravos de Notificação (SINAN).

\section{Resultados}

No Rio de Janeiro, no período de 2008 a 2017 foram registrados 790.505 nascidos vivos, 22.085 gestantes foram notificadas com sífilis na gestação, representando uma incidência da doença de 27,94 por mil nascidos vivos, conforme pode ser visualizado na série histórica do gráfico I.

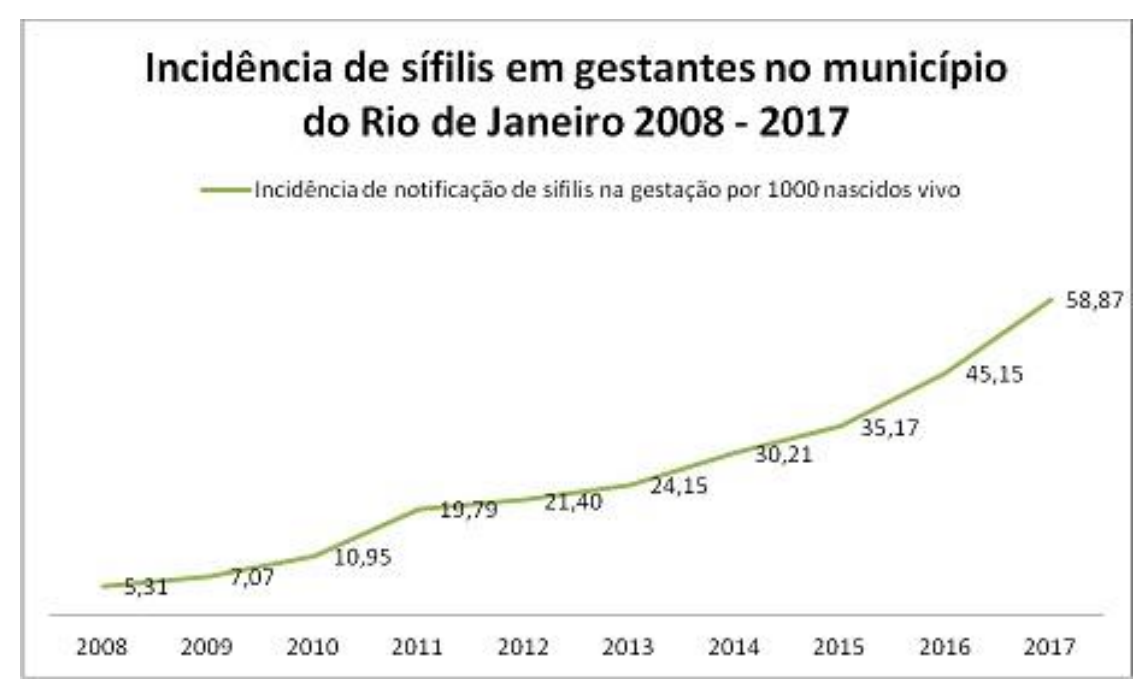

Gráfico 1 - Incidência anual de sífilis em gestante no município do Rio de Janeiro 2008-2017. 
Tabela I - Distribuição das características sociais das gestantes notificadas com sífilis no município do Rio de Janeiro 2008-2017.

\begin{tabular}{lll}
\hline Faixa etária & Total & Proporção \\
\hline $15-24$ & 12955 & $58,82 \%$ \\
$25-34$ & 6957 & $31,59 \%$ \\
$35-44$ & 1719 & $7,81 \%$ \\
$45-54$ & 31 & $0,14 \%$ \\
\hline Cor & Total & Proporção \\
\hline Ignorado & 3279 & $14,85 \%$ \\
Branca & 4466 & $20,22 \%$ \\
Preta & 4658 & $21,09 \%$ \\
Amarela & 230 & $1,04 \%$ \\
Parda & 9421 & $42,66 \%$ \\
\hline Escolaridade & Total & Proporção \\
\hline Ignorado & 6717 & $31,13 \%$ \\
Analfabeto & 278 & $1,29 \%$ \\
$1^{\text {1a a 4 }}$ a série incompleta & 1336 & $6,19 \%$ \\
$4^{\text {a }}$ série completa & 1127 & $5,22 \%$ \\
$5^{\text {a }}$ a 8 8 $^{\mathrm{a}}$ série incompleta & 3851 & $17,85 \%$ \\
Ensino fundamental completo & 2324 & $10,77 \%$ \\
Ensino médio incompleto & 2890 & $13,39 \%$ \\
Ensino médio completo & 2727 & $12,64 \%$ \\
Educação superior incompleta & 193 & $0,89 \%$ \\
Educação superior completa & 92 & $0,43 \%$ \\
\hline Fonte: Adaptado Tabnet-Datasus. 2018 & &
\end{tabular}

A tabela I proporciona analisar que a maioria das gestantes notificadas com sífilis são pardas e pretas $(63,75 \%)$, com faixa etária entre 15 e 24 anos $(58,82 \%)$ e com escolaridade igual ou inferior ao ensino médio completo (44,28\%), semelhante aos dados do Brasil [3].

Tabela II - Distribuição das características da assistência ao pré-natal das gestantes notificadas com sífilis no município do Rio de Janeiro 2008-2017.

\begin{tabular}{|c|c|c|}
\hline \multicolumn{3}{|c|}{ Período do diagnóstico da sífilis na gestação } \\
\hline & $\mathbf{N}$ & $\%$ \\
\hline 10 Trimestre & 8684 & $39,32 \%$ \\
\hline $2^{\circ}$ Trimestre & 5133 & $23,24 \%$ \\
\hline 3o Trimestre & 5784 & $26,19 \%$ \\
\hline Idade gestacional ignorada & 2232 & $10,11 \%$ \\
\hline Ign/Branco & 251 & $1,14 \%$ \\
\hline \multicolumn{3}{|c|}{ Esquema de tratamento da sífilis na gestação } \\
\hline & $\mathbf{N}$ & $\%$ \\
\hline Penicilina G benzantina $2.400 .000 \mathrm{UI}$ & 1267 & $5,74 \%$ \\
\hline Penicilina G benzantina $4.800 .000 \mathrm{UI}$ & 275 & $1,25 \%$ \\
\hline Penicilina G benzantina 7.200.000 Ul & 17362 & $78,61 \%$ \\
\hline Outro esquema & 170 & $0,77 \%$ \\
\hline Não realizado & 1254 & $5,68 \%$ \\
\hline $\operatorname{lgn} /$ Branco & 1759 & $7,96 \%$ \\
\hline \multicolumn{3}{|c|}{ Tratamento do parceiro realizado? } \\
\hline Parceiro Tratado & $\mathbf{N}$ & $\%$ \\
\hline SIM & 7213 & $32,66 \%$ \\
\hline NÃO & 5888 & $26,66 \%$ \\
\hline Ignorado & 8984 & $40,68 \%$ \\
\hline
\end{tabular}

A tabela II proporciona analisar o período de diagnóstico da sífilis nas gestantes, o tratamento prescrito pela equipe de pré-natalistas e a realização do tratamento pelo parceiro. Os dados apresentados evidenciam que a maior parte das gestantes são diagnosticadas no $1^{\circ}$ trimestre de gestação, realizam o tratamento com 7.200.000ui de penicilina G benzatina e $67,34 \%$ dos parceiros das gestantes não realizaram o tratamento ou não se tem esta informação. 
A incidência de casos notificados em gestante com sífilis no município do Rio de Janeiro vem crescendo com o passar dos anos. Este estudo evidencia que em 2008 o município apresentava uma incidência de 5,31/1000 nascidos vivos e em 2017 a incidência aumentou para 58,87 mulheres por 1000 nascidos vivos. É possível que o crescimento anual das notificações deu-se, em virtude da implantação da Resolução SS no 41 de 24/03/2005, que refere que deverá ser oferecido teste não treponêmico (VDRL) na primeira consulta de pré-natal para todas as gestantes, idealmente no primeiro trimestre de gestação e no início do terceiro trimestre, como também sua repetição para um controle da terapêutica no momento da internação para o parto ou curetagem, conforme recomendado pelo Ministério da Saúde [7].

Em relação as gestantes, a faixa etária predominante foi entre 15 e 24 anos, não brancas e com baixa escolaridade apresentado o maior percentual de infecção por sífilis na gestação. Segundo o Ministério da Saúde [7], a baixa faixa etária pode ser justificada pela vulnerabilidade da população com esta idade, que apresentam mais expostos às doenças sexualmente transmissíveis, visto que é uma fase de imaturidade etária, emocional e cognitiva, além de ser um período de constantes descobertas e de grande influência de grupos sociais.

Mascarenha [8] apresenta em seu estudo realizado com 90 adolescentes com idade entre 14 e 16 anos, estudantes da rede pública e privada, que a prática sexual entre os adolescentes está cada vez mais precoce e acompanhada da negligência no uso de contraceptivos tanto para evitar uma gravidez não planejada quanto na prevenção de doenças sexualmente transmissíveis [8].

Características, como cor não branca e baixa escolaridade são variáveis que se mostraram estatisticamente associadas à sífilis gestacional. Este é o perfil de indivíduos com uma condição socioeconômica menos favorecida e com menos acesso à saúde de qualidade. Contudo, não se pode afirmar que a sífilis seja uma condição de risco exclusivamente de populações mais carentes, ao contrário, independentemente da condição social ou econômica, todos podem adquirir a infecção, porém, o risco é em populações mais vulneráveis, tais condições podem estar associadas ao acesso à educação em saúde [9].

O presente estudo revela que apenas $39,32 \%$ das gestantes são diagnosticadas com sífilis no primeiro trimestre de gestação e o início tardio da assistência pré-natal é uma das barreiras para o controle da sífilis na gestação, segundo o Ministério da Saúde [7]. Esse início tardio associa-se ao menor número de consultas e menor tempo para o tratamento adequado da sífilis na gestação.

Para que a gestante seja considerada tratada adequadamente, afastando a possibilidade de infecção do concepto, deve ser medicada com penicilina $G$ benzatina, nas doses apropriadas na fase da infecção, ter finalizado o tratamento com pelo menos 30 dias antes do parto, e ter 0 parceiro concomitantemente tratado com o mesmo esquema terapêutico da gestante [10].

Em relação ao tratamento, o estudo mostra que em $78,61 \%$ das gestantes tem 0 tratamento prescrito com 7.200.000Ul de penicilina G benzantina, observado o recomendado pelo Ministério da Saúde [7]. Das gestantes em estudo, 21,39\% não realizaram ou não se tem informação do tratamento, o que torna um grave problema na saúde pública. Neste contexto é recomendado capacitar e sensibilizar os profissionais de saúde quanto à importância do tratamento adequado em todas as gestantes assistidas. Nos casos de gestantes comprovadamente alérgicas à penicilina, recomenda-se a dessensibilização, em serviço terciário de acordo com protocolos existentes. Os tratamentos não penicilínicos são inadequados e só devem ser considerados como opção nas contradições absolutas ao uso da penicilina [7].

Quanto ao tratamento do parceiro, o estudo aponta que apenas $32,66 \%$ realizaram o tratamento concomitante a gestante, $67,34 \%$ não realizaram ou não se tem a informação do tratamento.

A dificuldade de tratamento do parceiro sexual de portadores de DST pode estar relacionada à própria construção histórica das políticas de saúde, que sempre foram excludentes em relação ao homem, apresentando a baixa procura por atendimento. Pode também estar associada à visão que os homens têm em relação à saúde, pois atribuem à mulher a responsabilidade pelo cuidado [10].

Domingues [5] aponta que pelo menos $47 \%$ dos profissionais de saúde apresentam alguma dificuldade para realizar a abordagem ao parceiro para o tratamento da sífilis. Observase também que a falta de informação sobre o risco da doença e conhecimento sobre formas de prevenção e contágio da sífilis vem contribuindo para o aumento da incidência da doença. 
Portanto, há necessidade de maiores esclarecimentos à população sobre a doença, as formas de transmissão, prevenção e a importância do tratamento correto.

É referido que nas gestantes torna-se ainda mais complicado a infecção ou reinfecção da sífilis devido ao risco de contágio do concepto que fica exposto ao Treponema Pallidium já que esta bactéria traspõe a barreira trasplacentaria [8].

Considerando a incidência de sífilis em gestantes no município do Rio de Janeiro, o estudo mostra a importância dos profissionais de saúde na condução do controle e prevenção da doença, tendo em vista o perfil epidemiológico das gestantes com sífilis diagnosticada.

\section{Conclusão}

A pesquisa evidenciou que o perfil epidemiológico de mulheres notificadas com sífilis na gestação no período de 2008 a 2017 no município do Rio de Janeiro são mulheres com faixa etária entre 15 e 24 anos, pardas, com escolaridade inferior ao ensino fundamental, apresentando maior índice de notificação de sífilis no segundo e terceiro trimestre de gestação o tratamento realizado foi com 3 doses de penicilina G benzantina. Em relação ao tratamento dos parceiros sexuais, na maioria dos casos não há essa informação.

A limitação deste estudo deu-se em virtude de as variáveis apresentarem classificações como "Ignorado", que sugere prováveis sub-registro das notificações dos casos. Dessa forma cabe salientar que é necessário capacitar e sensibilizar os profissionais de saúde quanto a importância da qualificação do registro durante a notificação.

A partir dos dados apresentados nota-se que é essencial que os profissionais de saúde e a comunidade se sensibilizem sobre a importância do diagnóstico precoce e do tratamento eficaz da mulher e de seu parceiro, principalmente durante a gestação.

É importante a busca ativa das gestantes faltosas nas consultas de pré-natal, ações para a conscientização da população quanto aos riscos da prática sexual insegura e da importância do autocuidado, principalmente entre os mais vulneráveis.

1. Domingues RMSM, Leal MC. Incidência de sífilis congênita e fatores associados à transmissão vertical da sífilis: dados do estudo nascer no Brasil. Cad Saúde Pública Junho 2016;32. https://doi.org/10.1590/0102-311X00082415

2. Garcia FL. Prevalência de sífilis em adolescentes e jovens do sexo feminino no estado de Goiás [Dissertação]. Goiana: Universidade Federal de Goiás, Instituto de Patologia Tropical e Saúde Pública; 2009.

3. Ministério da Saúde. Boletim epidemiológico de sífilis. 2012. Disponível em: http://www.aids.gov.br/pt-br/pub/2017/boletim-epidemiologico-de-sifilis-2017

4. Secretária Municipal de Saúde do Município do Rio de Janeiro. Boletim epidemiológico DST/AIDS. 2013. Disponível em: http://www.rio.ri.gov.br/dlstatic/10112/4502878/4114721/anexo1 boletim.indd.pdf

5. Domingues RMSM, Saraceni V, Zulmira MA, Leal MC. Sífilis congênita: evento sentinela da qualidade da assistência pré-natal. Rev Saúde Pública 2013;47(1):147-57. https://doi.org/10.1590/S0034-89102013000100019

6. Costa MFL, Barreto SM. Tipos de estudos epidemiológicos: conceitos básicos e aplicações na área do envelhecimento. Epidemiol Serv Saúde 2003;12(4):189-201. https://doi.org/10.5123/S1679-49742003000400003

7. Ministério da Saúde. Protocolo clínico e diretrizes terapêuticas para atenção integral às pessoas com infecções sexualmente transmissíveis [Internet]. Brasília: Ministério da Saúde; 2015. Disponível em: http://www.aids.gov.br/publicacao/2015/protocolo-clinicoe-diretrizes-terapeuticas-para-atencao-integral-pessoas-com-infecc

8. Mascarenha LEF, Araujo MSS, Gramacho RCCV. Desafios no tratamento da sífilis gestacional. Repositório Bahiano 2016. Disponível em: http://www7.bahiana.edu.br//jspui/handle/bahiana/735

9. Rufino EC, Andrade SSC, Leadebal ODCP, Brito KKG, Silva FMC, Santos SH. Women's knowledge about STI/Aids: working with health education. Cienc Cuid Saúde 2016;15(2):304-11. Disponível em: http://periodicos.uem.br/ojs/index.php/CiencCuidSaude/article/view/26287 
10. Campos ALA, Maria ALA, Simone PM, Roumayne FV Andrade, Marcelo LCG. Sífilis em parturientes: aspectos relacionados ao parceiro sexual. Rev Bras Ginecol Obstet 2012;34(9):397-402. https://doi.org/10.1590/S0100-72032012000900002 